\title{
Búsqueda de señal orbital en isótopos estables de carbono y oxígeno de belemnites del Jurásico Inferior de la cuenca Vasco-Cantábrica mediante el periodograma suavizado de Lomb-Scargle
}

\section{Looking for orbital signals in stable isotopes of carbon and oxygen of belemnites from the Early Jurassic in the Basque Cantabrian basin through the smoothed Lomb-Scargle periodogram}

\author{
Josué M. Polanco-Martínez ${ }^{1,2}$ \\ 1*Basque Centre for Climate Change (BC3), 48940 Leioa, España. Email: josue.m.polanco@gmail.com. ORCID: https://orcid. \\ org/0000-0001-7164-0185 \\ ${ }^{2} \mathrm{IME}$, Universidad de Salamanca, 37007 Salamanca, España.
}

\begin{abstract}
The use of time series of stable isotopes obtained from fossilized samples of macrofossils, such as belemnites from the early Jurassic, until now, have not been used to explore whether these paleoindicators may have recorded some kind of periodic events related with the orbital cycles of the Earth. This, despite the fact that they are one of the few existing geological records for the early Jurassic. For this reason, and taking into account several of the limitations of these types of data sets, in this work, it is used a powerful spectral analysis technique known as the smoothed Lomb-Scargle periodogram, which can be applied directly to non-equispaced time series contaminated by noise. Furthermore, this work takes into account the dating errors of the belemnite samples in the estimation of the spectrum. The objective of this paper is to identify the presence of orbital cycles that have been recorded in time series of $\delta^{18} \mathrm{O}$ and $\delta^{13} \mathrm{C}$ from samples of belemnites collected in the Basque-Cantabrian basin. Special emphasis is placed on the search for the one of the eccentricity cycles, such as the long-period eccentricity cycle located ca. $400 \mathrm{Ka}$ or the short-period cycle located ca. $100 \mathrm{Ka}$, since the $400 \mathrm{Ka}$ cycle is known to have remained unchanged from the Mesozoic to the present and since both cycles have been identified in stratigraphic records of the Mesozoic collected in different geographic areas. However, despite the rigorousness of our analyzes, the long-period signal was not found, although we have located one ca. $109 \mathrm{Ka}$, which might be related with one the of cycles of short-period of the eccentricity of the Earth.
\end{abstract}

Keywords: Lomb-Scargle smoothed periogram; orbital cycles; stable isotopes; belemnites; early Jurassic.

Recibido el 2 de febrero de 2021; Aceptado el 24 de mayo de 2021; Publicado online el 24 de junio de 2021

Citation / Cómo citar este artículo: Polanco-Martínez, J.M. (2021). Búsqueda de señal orbital en isótopos estables de carbono y oxígeno de belemnites del Jurásico Inferior de la cuenca Vasco-Cantábrica mediante el periodograma suavizado de LombScargle . Estudios Geológicos 77(1): e140. https://doi.org/10.3989/egeol.44231.595

Copyright: @ 2021 CSIC. This is an open-access article distributed under the terms of the Creative Commons Attribution- Non Commercial (by-nc) Spain 4.0 License. 


\section{RESUMEN}

Los isótopos estables obtenidos de muestras fosilizadas de macrofósiles, como los belemnites, son unos de los pocos registros geológicos existentes del Jurásico Inferior, por lo cual han sido usados para reconstrucciones de temperaturas y salinidades del océano de esa época. Sin embargo, hasta la fecha no han sido utilizados para averiguar si estos paleoindicadores pudieron registrar algún tipo de evento periódico relacionado con los ciclos orbitales de la Tierra. Por esta razón y teniendo en cuenta varias de las limitaciones al utilizar estos tipos de datos, en este trabajo se presenta un novedoso estudio donde se aplica una técnica avanzada de análisis espectral conocida como el periodograma suavizado de Lomb-Scargle, la cual puede ser aplicada de modo directo a series temporales no equiespaciadas contaminadas por ruido. El objetivo de este trabajo es identificar la presencia de ciclos orbitales que se hayan podido registrar en series temporales de valores medios de $\delta^{18} \mathrm{O}$ y $\delta^{13} \mathrm{C}$ provenientes de muestras fosilizadas de belemnites recolectadas en la cuenca Vasco-Cantábrica teniendo en cuenta los errores de datación de estas muestras en la estimación del espectro. De manera especial se hace énfasis en la búsqueda de alguno de los ciclos de la excentricidad, como el de largo periodo localizado ca. $400 \mathrm{Ka}$ o el de menor periodo localizado ca. $100 \mathrm{ka}$, porque se sabe que el ciclo de $400 \mathrm{Ka}$ ha permanecido invariable desde el Mesozoico hasta el presente y porque ambos ciclos han sido identificados en registros estratigráficos del Mesozoico recolectados en diferentes áreas geográficas. Sin embargo, a pesar de la rigurosidad de nuestros análisis, no se encontró señal orbital de $400 \mathrm{Ka}$ aunque si una localizada cerca de los 109 Ka, la cual podría estar relacionada con los ciclos de corto periodo de la excentricidad de la Tierra.

Palabras clave: Periodograma suavizado de Lomb-Scargle; ciclos orbitales; isótopos estables; belemnites; Jurásico Inferior.

\section{Introducción}

El Jurásico Inferior (Plienbachiense y Toarciense Inferior), datado entre los 182-190 Ma, ha llamado mucho la atención de la comunidad científica debido a que fue una época de grandes cambios y crisis ambientales. Existió un breve intervalo de calentamiento global (se estima que la temperatura promedio del aire en superficie estuvo entre 5 y $10{ }^{\circ} \mathrm{C}$ por encima de la temperatura promedio actual de la Tierra), un aumento del nivel eustático del mar, alta actividad volcánica, liberación de grandes cantidades de metano, aumento excepcional en la depositación de material con alto contenido de carbono orgánico a nivel global debido en parte como resultado de varios eventos oceánicos anóxicos (Oceanic Anoxic Event, OAE) y extinciones masivas de la biodiversidad (Hesselbo et al., 2000; Bailey et al., 2003; Suan et al., 2008; Hermoso et al., 2012; Kemp \& Izumi, 2014; Bodin et al., 2016; Remírez \& Algeo, 2020). Estos cambios ambientales han sido registrados en diversos materiales geológicos provenientes de distintas cuencas alrededor del mundo (Hesselbo et al., 2000; Jenkyns et al., 2002; Kemp et al., 2005; Rosales et al., 2004a; Suan et al., 2008; Hermoso et al., 2012; Bodin et al., 2016). Una gran cantidad de estudios han sido realizados para saber la causa de estos cambios, y si los mismos se produjeron a escala global (Bailey et al., 2003; Suan et al., 2008; Bodin et al., 2016; Remírez \& Algeo, 2020). Sin embargo, a pesar de estos esfuerzos, no existe una sola respuesta para explicarlos. Por ejemplo, no se conoce con exactitud el papel de las señales orbitales en el ciclo del carbono, como en las excursiones del $\delta^{13} \mathrm{C}$ asociados con el OAE del Toarciense Inferior (Suan et al., 2008; Storm et al., 2020).

Una forma de estudiar los cambios ambientales del pasado consiste en analizar las relaciones geoquímicas de isótopos estables de carbono y oxígeno de material fosilizado de macrofósiles, como las rostra de belemnites. Esta especie extinta que vivió en el Jurásico y Cretácico ha sido considerada como uno de los grupos de fósiles más adecuados para estimaciones de paleotemperaturas y paleosalinidades del océano de esa época (Podlaha et al., 1998; McArthur et al., 2000; Jenkyns et al., 2002; McArthur et al., 2007; Gómez et al., 2008; Armendáriz et al., 2013; Gómez et al., 2016; Arabas et al., 2017). Con lo que respecta al área geográfica de este estudio, i.e., la cuenca Vasco-Cantábrica (Basque-Cantabrian Basin, BCB), Rosales et al. (2001, 2004a, 2004b, 2006) propusieron que valores medios del $\delta^{18} \mathrm{O}$ y $\delta^{13} \mathrm{C}$ provenientes de las rostra de belemnites podrían utilizarse a escala local o regional para hacer estimaciones de paleotemperaturas y paleosalinidades del océano, reconstrucciones de la circulación oceánica, paleo-productividad y determinar cambios en el nivel del mar durante el Jurásico Inferior. Estos estu- 
dios también sugieren que parte de la variabilidad de los valores medios del $\delta^{18} \mathrm{O}$ y $\delta^{13} \mathrm{C}$ podrían reflejar algún tipo de señal orbital, como los ciclos de precesión (ca. $20 \mathrm{Ka})$, oblicuidad (ca. $40 \mathrm{Ka})$ o excentricidad (ca. $100 \mathrm{Ka}$ o $400 \mathrm{Ka}$ ), tal y como se han encontrado en otros paleoindicadores (e.g., $\mathrm{CaCO}_{3}$, TOC, susceptibilidad magnética, entre otros) ambientales del Plienbachiense y Toarciense en diferentes zonas geográficas cercanas a la cuenca Vasco-Cantábrica (Hinnov \& Park, 1999; Weedon et al., 1999; Weedon \& Jenkyns, 1999; Suan et al., 2008; Kemp et al., 2011; Bádenas et al., 2012; Storm et al., 2020).

Una herramienta adecuada para la búsqueda de ciclos orbitales en series temporales paleoclimáticas es el análisis espectral (Schulz \& Stattegger, 1997; Weedon, 2003; Pardo-Iguzquiza \& Rodríguez-Tovar, 2011, 2012, 2013; Medina-Elizalde et al., 2016; Oliveira et al., 2017). Esta búsqueda es relativamente sencilla cuando las series provienen de material fosilizado con alta resolución espacio-temporal de muestreo (como las series de $\mathrm{CaCO}_{3}$ ) (Weedon \& Jenkyns, 1999; Weedon, 2003). Sin embargo, esta búsqueda es más complicada cuando las series provienen de material marino calcáreo fosilizado (como las rostra de belemnites), porque existe un cierto grado de aleatoriedad en la localización espacial de las muestras, lo cual a su vez es debido al modo de vida de los belemnites (Podlaha, et al., 1998; Weedon, 2003; Rexfort \& Mutterlose, 2006; Nava, 2010). Por esta razón, las series temporales creadas con estos materiales no suelen estar equiespaciadas temporalmente, incluso, pueden presentar grandes diferencias espacio-temporales entre las muestras (Weedon, 2003; Nava, 2010).

Una posible solución para enfrentar el carácter no equiespaciado de las series temporales paleoclimáticas es interpolar en el tiempo y, así, obtener una serie temporal equiespaciada. Sin embargo, la interpolación implica un conocimiento previo del comportamiento de la variable de estudio, y suaviza los datos de tal modo que al calcular el espectro puede suprimir información espectral en las altas frecuencias (Schulz \& Stattegger, 1997; Schulz \& Mudelsee, 2002; Pardo-Iguzquiza \& Rodríguez-Tovar, 2012; Polanco-Martínez, 2014; Polanco-Martínez \& Faria, 2018). Otra solución consiste en utilizar métodos de análisis espectral que puedan aplicarse directamente (sin previa interpolación en el tiempo) a las series temporales paleoclimáticas, tales como el periodograma suavizado de Lomb-Scargle (Lomb 1976; Scargle 1982, 1989; Schulz \& Stattegger, 1997; Schulz \& Mudelsee, 2002; Pardo-Igúzquiza \& Rodríguez-Tovar 2011; 2012; 2013; Polanco-Martínez, 2014). No obstante, en algunos casos, como en el de las series temporales de los valores isotópicos utilizadas en este estudio, las diferencias entre los tiempos de una serie temporal son mucho más grandes que la escala de las mayores oscilaciones de la señal que se espera encontrar. Entonces, ni la interpolación ni los métodos especiales de análisis espectral pueden ser de mucha ayuda (Weedon, 2003; Polanco-Martínez, 2012). Una posible solución consiste en partir la serie original en trozos más cortos, utilizando como punto de corte los tiempos que superan las mayores oscilaciones esperadas (Weedon, 2003).

Otro factor que puede complicar el análisis espectral de series temporales cortas y no equiespaciadas temporalmente, como las series de $\delta^{18} \mathrm{O}$ y $\delta^{13} \mathrm{C}$ de los belemnites en este estudio, es la presencia de ruido. Se sabe que los paleoindicadores ambientales provenientes de belemnites (e.g., $\delta^{18} \mathrm{O}$ y $\left.\delta^{13} \mathrm{C}\right)$ contienen ruido (Podlaha, et al., 1998; McArthur et al., 2007). Este ruido puede representar cambios en los procesos ambientales ocurridos en el pasado (frecuentemente de corto periodo) en especies de belemnites adaptables a estos cambios (i.e., especies que no hayan migrado como una respuesta a estos cambios). Sin embargo, también pueden reflejar el rango espacial, vertical (profundidad oceánica) y lateral (geográfico) del hábitat de los belemnites, incluso pueden reflejar el efecto vital de las especies, del género o la edad biológica del belemnite (Podlaha, et al., 1998; McArthur et al., 2007).

Teniendo en consideración varias de las características de las series temporales de paleoindicadores provenientes de los belemnites del jurásico Inferior. Este trabajo tiene el objetivo de averiguar si los valores medios del $\delta^{18} \mathrm{O}$ y del $\delta^{13} \mathrm{C}$ de los belemnites de la cuenca Vasco-Cantábrica (Rosales et al., 2001, 2004a, 2004b) pudieron haber registrado algún tipo de evento periódico relacionado con los ciclos orbitales de la Tierra a pesar del ruido contenido en estos isótopos estables y a las limitaciones impuestas por la componente estocástica en el muestreo de los be- 
lemnites. Cabe mencionar que hasta donde se sabe, es la primera vez que se analizan series temporales en el dominio de la frecuencia de valores medios de $\delta^{18} \mathrm{O}$ o de $\delta^{13} \mathrm{C}$ obtenidos de la rostra fosilizada de belemnites. Especial énfasis se hace en la búsqueda de los ciclos de excentricidad de largo periodo localizado $c a .400 \mathrm{Ka}$ y el de más corto periodo $c a .100$ Ka (Berger \& Loutre, 2004; Laskar et al., 2004; Crucifix et al., 2006; Laskar et al., 2011), porque se sabe que el ciclo de los $400 \mathrm{Ka}$ ha permanecido invariable desde el Mesozoico hasta el presente (Laskar et al., 2004; Hinnov \& Ogg, 2007; Laskar et al., 2011) y porque estos dos ciclos han sido identificados en registros estratigráficos del Mesozoico recolectados en diferentes áreas geográficas (Olsen, 1986; Weedon, 1989; Olsen \& Kent, 1996, Olsen \& Kent 1999; Weedon et al., 1999; Weedon \& Jenkyns, 1999; Suan et al., 2008; Kemp et al., 2011; Storm et al., 2020).

Para poder llevar a cabo este objetivo, se ha utilizado una técnica no estándar de análisis espectral conocida como el periodograma suavizado de Lomb-Scargle (Lomb, 1976; Scargle, 1982, 1989). Este técnica se aplica de modo directo a series temporales no equiespaciadas en el tiempo y está implementado en el paquete computacional REDFIT (Schulz \& Mudelsee, 2002), el cual tiene en cuenta el tipo de ruido de fondo ("rojo") de las series (paleo) climáticas (Gilman et al., 1963; Hasselmann, 1976; Schulz \& Mudelsee, 2002; Weedon, 2003). Existen también otros paquetes computacionales para estimar el espectro suavizado mediante el periodograma de Lomb-Scargle, tal como SLOMB (Pardo-Iguzquiza \& Rodríguez-Tovar, 2011, 2012). En este trabajo también se presenta una novel y sencilla aproximación para tener en cuenta la componente aleatoria en la estimación del espectro debido a los errores de datación de las muestras de belemnites.

\section{Material y métodos}

\section{Marco geológico}

Hace unos 183 millones de años la distribución física (continentes y océanos) de la Tierra era muy diferente a la de hoy en día. De acuerdo a reconstrucciones paleocontinentales realizadas por Ziegler (1982) y Baudin et al. (1990) para el Jurásico Infe- rior, la cuenca Vasco-Cantábrica (Figura 1A) estuvo localizada en un mar epicontinental, limitada al norte por el macizo Armoricano y al sur por el macizo Ibérico, y conectaba el mar Boreal del norte con el océano Tethys del sur (Decourt 1993; Rosales et al 2004a). Las muestras fosilizadas de rostra de belemnites fueron colectadas por Rosales et al. (2001, 2004a, 2004b) en la cuenca Vasco-Cantábrica, norte de España (Figura 1 B) y aunque consisten de unas 200 muestras, éstas se redujeron a 176 debido a que son las que no presentaron efectos diagenéticos (Rosales et al. 2001, 2004a, 2004b). Estas muestras forman una sección compuesta que abarca 9.6 Ma (de 191.60 a $182.00 \mathrm{Ma}$ ) y pertenecen principalmente al Plienbachiense (90\% de los datos) y al Toarciense Inferior (Figura 1B). El muestreo fue realizado por Rosales et al. (2004a) de acuerdo con Braga et al. (1988) y Comas-Rengifo et al. (1988) en intervalos estratigráficos de $0.2-5 \mathrm{~m}$ y fue calibrado contra bioestratigrafía disponible basada en biozonas de amonites. La edad de las muestras en la misma sucesión fue estimada mediante análisis bioestratigráfico por Rosales et al. (2004a) y está basada en la duración de cada zona y sub-zona de amonites (considerando la tasa de sedimentación constante para cada una de ellas) calculadas desde la razón de cambio del ${ }^{87} \mathrm{Sr} /{ }^{86} \mathrm{Sr}$ con edades basadas en McArthur et al. (2000, 2001) y como tie points edades radiométricas de Pálfy et al. (2000). Para más detalles sobre la cronología puede consultarse Rosales et al. (2004a).

\section{Series temporales de valores medios de $\delta^{18} \mathrm{O} y$} $\delta^{13} \mathrm{C}$

En las figuras 2A y 2B se presentan las series temporales de los valores medios (se usan valores medios porque en una misma capa pueden haber varias partes fosilizadas de un rostrum de belemnites o varias muestras de diferentes especies de belemnites) de $\delta^{18} \mathrm{O}$ (Rosales et al., 2004a) y de $\delta^{13} \mathrm{C}$ (Rosales et al., 2001, 2004b) provenientes de las muestras de belemnites de la cuenca Vasco Cantábrica. Las áreas grises representan eventos oceánicos anóxicos (OAE) y las líneas verticales discontinuas indican el intervalo temporal donde se aplica el análisis espectral. Debido a que hay muy pocas muestras de belemnites en las biozonas jamesoni y serpentinus, y 
para satisfacer la propiedad de estacionariedad, ambas series temporales han sido recortadas para formar una sección que va de 188.68 a $183.36 \mathrm{Ma}$. La sección abarca unos 5.3 Ma y contiene 81 elementos. Las series temporales de los valores medios de $\delta^{18} \mathrm{O}$ y $\delta^{13} \mathrm{C}$ no han sido interpoladas en el tiempo y no se
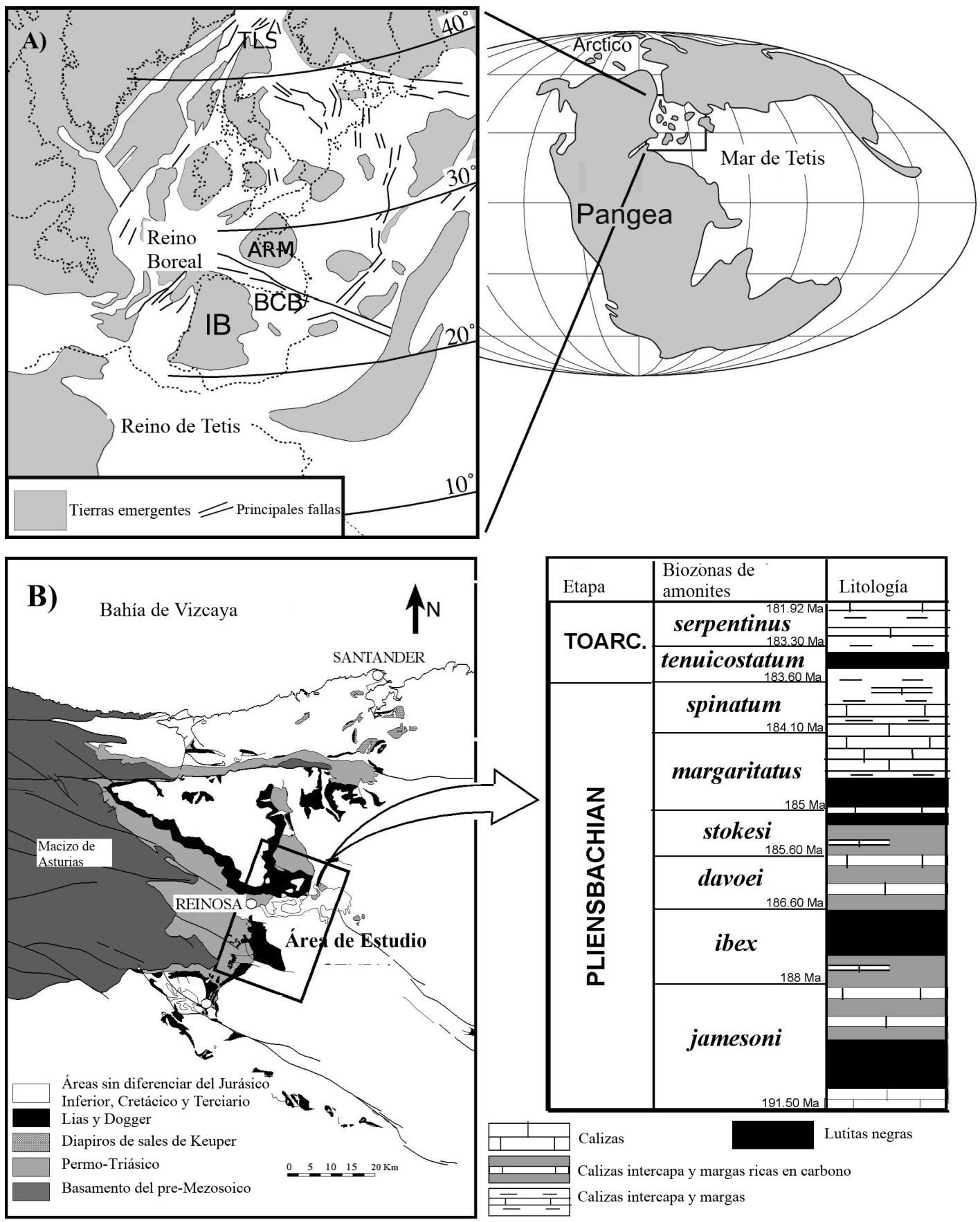

Figura 1-A) Paleogeografía del Jurásico Inferior de la región noroeste de Europa. Modificado a partir de Ziegler (1990) y Baudin et al. (1990). La región de estudio, la cuenca Vasco-Cantábrica (BCB), estuvo localizada entre 20 y $30^{\circ}$ latitud Norte. ARM y IB son los macizos Armoricano e Ibérico y TLS es el Transcontinental Laurasian Seaway. B) Representación geográfica-geológica y sección estratigráfica simplificada del área donde las rostra de belemnites fueron recolectadas, la BCB (Norte de España). Tomada y modificada de Rosales et al. (2004a). 
A)

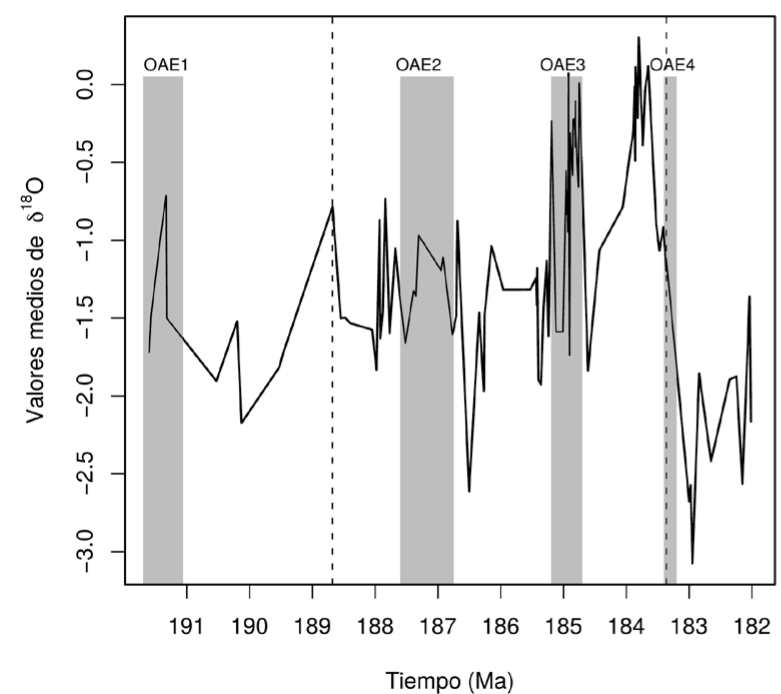

B)

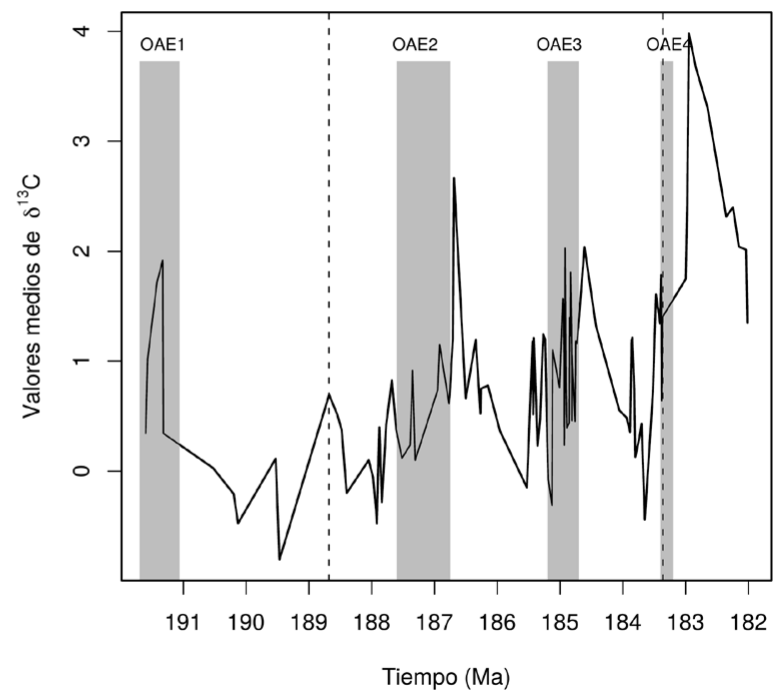

Figura 2- Series temporales no equiespaciadas en el tiempo de valores medios de $\delta^{18} \mathrm{O} \%$ PDB (A) (Rosales et al., 2004a) y de $\delta^{13} \mathrm{C} \%$ PDB (B) (Rosales et al., 2001, 2004b) de las rostra de belemnites del Jurásico Inferior. Las áreas grises representan eventos oceánicos anóxicos (OAE) y las líneas verticales discontinuas indican el intervalo temporal donde se aplica el análisis espectral $(\mathrm{N}=$ 81 datos). Fuente: elaboración propia.

ha aplicado ningún tipo de orbital tuning. La razón para ello es que el orbital tuning puede introducir razonamiento circular en el análisis de resultados y porque los periodos (ciclos) utilizados como objetivos para hacer el tuning, muchas veces están basados en propiedades espectrales de una serie de tiempo más que en los procesos físicos envueltos en su generación (Huybers \& Wunsch, 2004; Lisiecki, 2005; Blaauw, 2012).

\section{Análisis espectral mediante el Periodograma sua- vizado de Lomb-Scargle}

Las series temporales de los valores medios de $\delta^{18} \mathrm{O}$ y $\delta^{13} \mathrm{C}$ no están equiespaciadas en el tiempo. Para evitar la interpolación se utiliza una versión adaptada por Schulz \& Statteger (1997) y Schulz \& Mudelsee (2002) del periodograma de Lomb-Scargle (Lomb-Scargle Fourier Transform, LSFT) (Lomb, 1976; Scargle, 1982, 1989) para analizar series paleoclimáticas. Esta adaptación tiene en cuenta el tipo de ruido de fondo presente en las series temporales paleoclimáticas, i.e., ruido rojo (Gilman et al., 1963; Hasselmann, 1976; Schulz \& Mudelsee, 2002; Weedon, 2003) y lleva de nombre REDFIT (Schulz \& Mudelsee, 2002). REDFIT está disponible en https://www.marum.de/Binaries/Bi- nary2502/redfit38e.zip y también existe un paquete computacional en lenguaje $\mathrm{R}$ de nombre "dplR" que tiene integrado REDFIT, el cual está disponible en https://cran.r-project.org/package=dplR. Para discernir si un pico espectral de las series temporales a estudio es significativo contra la hipótesis de que ha sido producido por un ruido de fondo rojo, para lo cual se usa un modelo del tipo AR1 (proceso autorregresivo de orden 1), su espectro es comparado con el espectro teórico correspondiente al ruido rojo. Una descripción detallada de este método de análisis espectral puede encontrarse en Schulz \& Mudelsee (2002) o en Polanco-Martínez (2012, 2014). Los parámetros de entrada de REDFIT utilizados en este caso de estudio están resumidos en la Tabla 1.

La versión de REDFIT (3.5) que se ha utilizado en este trabajo no incorpora errores de datación, aunque la más reciente versión (Mudelsee et al., 2009) sí tiene en cuenta esta característica, pero no es de libre acceso. Por lo tanto, en este trabajo se ha tenido que implementar un programa computacional en lenguaje $\mathrm{R}$ en combinación con REDFIT 3.5 para tener en cuenta los errores de datación de las muestras de belemnites para lo cual se utilizan simulaciones de Monte-Carlo (este programa está disponible 
Tabla 1. Parámetros de entrada para estimar los espectros suavizados mediante REDFIT. a es el Intervalo promedio de muestreo, b es el Welch's Overlapped Segment Averaging (WOSA), ${ }^{c}$ son los grados de libertad (degrees of freedom o dof), $\mathrm{y}^{\mathrm{d}}$ es el coeficiente promedio de persistencia.

\begin{tabular}{|l|l|}
\hline Parámetro & Valor \\
\hline $\mathrm{N}$ (número de elementos) & 81 \\
Frecuencia mínima & fo $=1.17 \mathrm{e}-7$ \\
Frecuencia Máxima & Fmax $=1.5 \mathrm{e}-5$ \\
$<\Delta t>^{\mathrm{a}}$ & 66,450 años \\
Número de segmentos WOSA & $\mathrm{N} 50=4$ \\
Factor "oversampling" (sobremuestreo) & ofac $=4$ \\
Tipo de ventana & iwin $=$ Hanning \\
Número de simulaciones de Monte-Car- & 10,000 \\
lo & 8 \\
dof ${ }^{\mathrm{c}}$ & 35,000 años \\
$<\mathrm{T}_{\mathrm{T}}>\mathrm{d}$ & \\
\hline
\end{tabular}

Fuente: elaboración propia.

para cualquier persona interesada, por favor contacte con el autor por correspondencia). Los errores de datación pueden ser considerados como un proceso Gaussiano centrado en la mejor estimación de la datación de una muestra y con una desviación estándar $\delta_{\mathrm{d}}$ que depende de la abundancia relativa de los fósiles de belemnites dentro de cada capa. Este procedimiento puede ser considerado como una adaptación del modelo propuesto por Fleitmann et al. (2003) y por Mudelsee et al. (2009), y se resume en los siguientes pasos:

1) Sean $\{\mathrm{t}(\mathrm{i})\}_{\mathrm{i}=1}^{\mathrm{N}}$ los tiempos de las muestras de belemnites y $\{\mathrm{y}(\mathrm{i})\}_{\mathrm{i}=1}^{\mathrm{N}}$ los valores isotópicos.

2) Para $k=1$, Nsims
a. $\mathrm{t}^{*}(\mathrm{i})=\mathrm{t}(\mathrm{i})+\varepsilon_{\mathrm{N}(0, \delta \mathrm{d})}^{2}$, los tiempos perturba- dos, y donde $\delta \mathrm{d}=50 \mathrm{Ka}$

b. Se verifica que los nuevos tiempos $\mathrm{t}^{*}$ para cada realización $\mathrm{k}$, tengan un incremento estrictamente monótono.

c. Se calcula el espectro de $\left\{t^{*}(i), y(i)\right\}{ }_{i=1}^{N}$ mediante REDFIT 3.5

4) Se calculan los percentiles (95\%) de los Nsims espectros.

donde Nsims es el número de simulaciones de Monte-Carlo (10,000 en este estudio) y los valores de entrada de REDFIT están proporcionados en la Tabla 1. Nótese que cada vez que REDFIT estima un espectro para cada realización $\left\{\mathrm{t}^{*}(\mathrm{i}), \mathrm{y}(\mathrm{i})\right\}_{\mathrm{i}=1}^{\mathrm{N}}$, también estima su correspondiente nivel de ruido rojo con un nivel de confianza del 95\%.

\section{Resultados y discusión}

Los resultados obtenidos después de aplicar análisis espectral por medio del periodograma suavizado (con 8 grados de libertad) de Lomb-Scargle a los valores medios de $\delta^{18} \mathrm{O}$ y $\delta^{13} \mathrm{C}$ y teniendo en cuenta los errores de datación de las muestras de los belemnites se muestran en la Figura 3. Los picos espectrales que superan el espectro asociado a un ruido de tipo rojo con un nivel de confianza del $95 \%$ están localizados ca. 1.3 Ma para el $\delta^{18} \mathrm{O}$ y $c a .2 .1 \mathrm{Ma}$ y $c a .109 \mathrm{Ka}$ para el $\delta^{13} \mathrm{C}$.
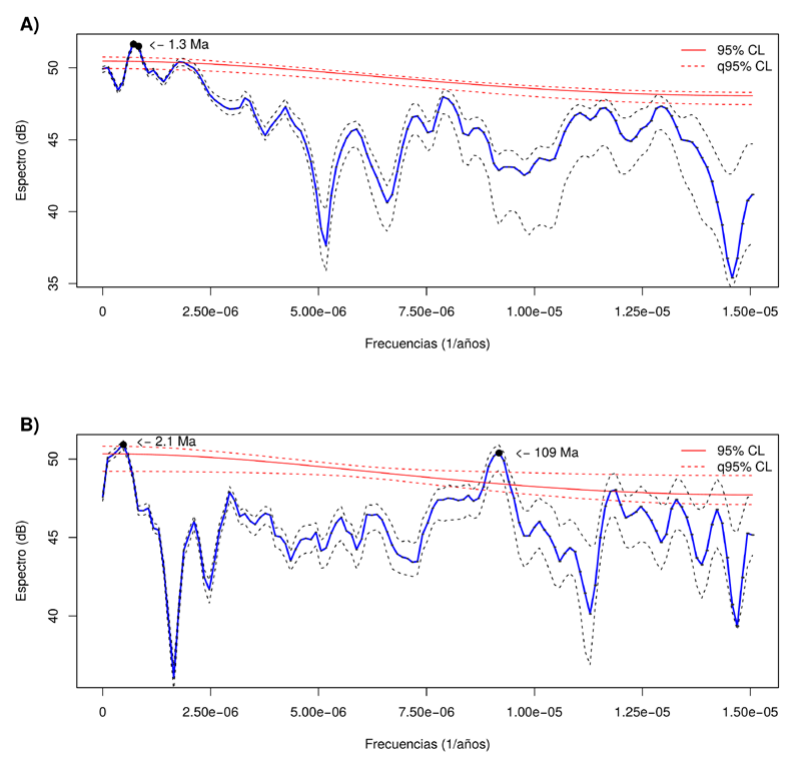

Figura 3- Espectros corregidos y suavizados (con 8 grados de libertad) de las series temporales de los valores medios de $\delta^{18} \mathrm{O}$ (A) y $\delta^{13} \mathrm{C}$ (B) (curvas continuas de color azul) para el intervalo 188.68-183.36 Ma. El espectro fue calculado por medio del programa REDFIT, los parámetros de entrada pueden consultarse en la Tabla 1. Las curvas discontinuas (en negro) que rodean el espectro, son los intervalos de confianza (5 y $95 \%$ ) del espectro al tener en cuenta los errores de datación mediante el método de Monte-Carlo. La curva sólida de color rojo representa el $95 \%$ del nivel de confianza correspondiente a ruido rojo. Las curvas discontinuas de color rojo, son los intervalos de confianza (5 y $95 \%$ ) del ruido al tener en cuenta los errores de datación de las muestras de belemenites. Se han utilizado 10,000 simulaciones de Monte-Carlo. Fuente: elaboración propia.

Los valores medios de $\delta^{18} \mathrm{O}$ y $\delta^{13} \mathrm{C}$ provenientes de carbonatos marinos, como las rostra de belemnites, pueden estar influenciados principalmente por los siguientes factores: procesos diagénicos, procesos fracciónales biogénicos, variaciones isotópicas intra-especimen e inter-especimen y factores ambien- 
tales (Podlaha et al., 1998; McArthur et al., 2000; Rosales et al., 2001; Rosales et al., 2004a; McArthur et al., 2007; Gomez et al., 2008, 2016; Immenhauser et al., 2016; Arabas et al., 2017). El primer factor en nuestro estudio, puede ser descartado como la principal causa, porque las muestras de belemnites utilizadas para obtener los valores isotópicos medios de $\delta^{18} \mathrm{O}$ y $\delta^{13} \mathrm{C}$ fueron previamente verificadas por Rosales et al. (2004a,b) ante posibles efectos de alteración diagénica. El grado de alteración diagénica de las belemenites fue realizado mediante la aplicación de técnicas petrográficas y de catodoluminisencia así como análisis geoquímico y fue llevado a cabo por Rosales et a. (2004b) siguiendo el trabajo de Rosales et al. (2001).

El segundo factor, i.e., los efectos vitales, son más difíciles de cuantificar porque los belemnites son organismos extintos (Rosales et al., 2001; Rexfort \& Mutterlose, 2006; McArthur et al., 2007; Benito et al., 2016; Immenhauser et al., 2016; Hoffmann \& Stevens, 2020). Sin embargo, a pesar de que en este trabajo no es posible abordar este punto, si deberían de ser tenidos en consideración al analizar paleoindicadores que provienen de partes fosilizadas de belemnites. Estudios previos han demostrado que las muestras de belemnites provenientes de diferentes lugares y edades no se agrupan isotópicamente de acuerdo a las especies, por ello, es poco probable que exhiban efectos vitales (Sælen et al., 1996; Rosales et al., 2001). Por otro lado, se sabe que los belemnites tenían cierto parecido a los actuales cefalópodos (Rosales et al., 2001; Wierzbowski, 2004; Rexfort \& Mutterlose, 2006; Benito et al., 2016; Hoffmann \& Stevens, 2020), y que, en estos últimos, los efectos vitales no influyen en la composición isotópica del oxígeno presente en sus conchas. En base a estos argumentos, Wierzbowski (2004) y Rexfort \& Mutterlose (2006) sugieren que no se espera encontrar efectos de biofraccionamiento isotópico en la rostra fosilizada de belemnites.

El tercer factor, $i$. e., las variaciones isotópicas intra-especimen e inter-especimen, son discutidas a continuación como posibles factores en el comportamiento de las series temporales de los isótopos estables analizados. Las series temporales en este estudio corresponden a valores medios de los isótopos estables $\delta^{18} \mathrm{O}$ y $\delta^{13} \mathrm{C}$, esto significa que para una misma capa puedan encontrarse diferentes secciones fosilizadas de un mismo belemnite o varias muestras de especies diferentes. Rosales et al. (2001) demostraron que las variaciones de los valores isotópicos $\left(\delta^{18} \mathrm{O}\right.$ y $\left.\delta^{13} \mathrm{C}\right)$ intra-especimen no superan un $0.3 \%$ para una misma capa, siempre y cuando las muestras no presenten efectos diagénicos. Por ello, no parecen jugar un papel importante en las variaciones de los valores isotópicos. Sin embargo, las variaciones de los valores isotópicos (191.60-182 Ma) inter-especimen, para una misma capa, son mucho mayores, hasta un $1.2 \%$ para el $\delta^{18} \mathrm{O}$ y hasta un $2.1 \%$ para el $\delta^{13} \mathrm{C}$. Rosales et al. (2001) sugieren que las variaciones de los valores isotópicos inter-especimen para una determinada capa, podrían reflejar el comportamiento paleo-ecológico de diferentes especies de belemnites o podrían estar causadas por variaciones seculares de alta frecuencia como los ciclos de Milankovitch ( $10^{4}-10^{5}$ años). Respecto a la primera hipótesis, Rosales et al. (2001) sugieren que diferentes especies de belemnites que hayan coexistido en diferentes hábitats, pueden presentar diferentes composiciones isotópicas de $\delta^{18} \mathrm{O}$ si durante sus ciclos de vida hayan habitado de modo preferente en diferentes masas de agua (aguas profundas y frías, aguas superficiales y cálidas). Aunque los belemnites son considerados organismos nectónicos, hay estudios que sugieren que existieron especies del tipo nectobentónico (Anderson et al., 1994; Price \& Sellwood, 1997; Rexfort \& Mutterlose, 2006; Hoffmann \& Stevens, 2020). Estos argumentos sugieren la importancia de identificar las especies de las muestras de belemnites en el intento de explicar las fluctuaciones de los valores isotópicos, sin embargo, para este estudio, desafortunadamente no se tiene disponible esa información. La segunda hipótesis no se puede descartar, aunque es difícil de sostener porque hay pocas muestras por cada biozona y debido a las restricciones crono y bio-estratigráficas no se puede resolver temporalmente más allá de una biozona de amonites (Rosales et al., 2001). Sin embargo, quizá es posible detectar algún tipo de ciclo orbital de más largo periodo que los de Milankovitch (periodo mayor que $10^{5}$ años) pero teniendo en cuenta varias biozonas, como en la serie de los valores isotópicos $\left(\delta^{18} \mathrm{O}\right.$ y $\delta^{13} \mathrm{C}$ ) analizada en este estudio.

El cuarto factor, la influencia de los factores paleo-ecológicos y ambientales, se discute a continua- 
ción. Es posible que los efectos paleo-ecológicos de los belemnites puedan influir en la composición isotópica (Rosales et al., 2001; Rexfort \& Mutterlose, 2006; McArthur et al., 2007; Benito et al., 2016; Immenhauser et al., 2016; Hoffmann \& Stevens, 2020). Es ampliamente aceptado que los belemnites fueron un tipo de organismo nectónico, esto quiere decir que muy probablemente hayan podido migrar lateral y verticalmente a través de masas de agua de diferente temperatura o salinidad (Doyle \& MacDonald, 1993; Podlaha et al., 1998; Rexfort \& Mutterlose, 2006; McArthur et al., 2007; Hoffmann \& Stevens, 2020). Si este fuera el caso, la composición isotópica de las rostra podría haber cambiado durante su crecimiento en respuesta a los cambios en el ambiente (e.g., temperatura o salinidad) (McArthur et al., 2007; Rexfort \& Mutterlose, 2006; Benito et al., 2016; Hoffmann $\&$ Stevens, 2020). Por otro lado, en trabajos previos Rosales et al. (2004a, 2004b) utilizaron exitosamente valores medios de $\delta^{18} \mathrm{O}$ para las reconstrucciones de paleotemperaturas y paleosalinidades. Esto quiere decir que las variaciones de los valores medios de $\delta^{18} \mathrm{O}$ están relacionadas, hasta cierto punto, con cambios en la temperatura/salinidad del océano de esa época (Rosales et al., 2004a, 2004b). En un trabajo más reciente, Rosales et al. (2006) muestran que los valores isotópicos de $\delta^{18} \mathrm{O}$ y $\delta^{13} \mathrm{C}$ obtenidas de las rostra de belemnites de la cuenca Vasco-Cantábrica muestran perturbaciones en las condiciones paleooceanográficas a nivel local y regional, como cambios en la temperatura, salinidad y circulación durante el Jurásico Inferior. Sin embargo, estos resultados no contemplan la posibilidad de que las variaciones isotópicas $\left(\delta^{18} \mathrm{O}\right.$ y $\left.\delta^{13} \mathrm{C}\right)$ pudieran haber sido influenciadas por forzamientos externos, tales como los ciclos orbitales de largo periodo. Para analizar esta posibilidad en este trabajo se emplean las estimaciones de los espectros suavizados de los valores medios del $\delta^{18} \mathrm{O}$ y $\delta^{13} \mathrm{C}$.

Los únicos picos espectrales significativos de largo periodo están localizados ca. 1.3 Ma para el $\delta^{18} \mathrm{O}$ (Figura $3 \mathrm{~A}$ ) y ca. $2.1 \mathrm{Ma}$ para el $\delta^{18} \mathrm{O}$ (Figura $3 \mathrm{~B}$ ). Sin embargo, a pesar de ser significativos y de tener en cuenta en el espectro los errores de datación, estos picos espectrales podrán estar influenciados por la tendencia de las series temporales. Es cierto que la tendencia lineal ha sido removida antes de calcular el espectro, pero también hay que tener en cuenta que el cálculo de la tendencia en una serie temporal es algo complicado, y es prácticamente imposible (en especial cuando no se conocen bien los procesos subyacentes del sistema que representan) separar de forma suficientemente precisa, tendencia, periodicidades de muy baja frecuencia y ruido (Van Dongen et al., 1999; Wu et al., 2007). Más aun, es complicado ubicar de forma precisa el ciclo orbital en una u otra frecuencia con una serie temporal tan corta. No obstante, suponiendo que 2.1 o 1.3 Ma representan eventos periódicos, entonces en la sección 188.68183.36 Ma puede haber a lo sumo 3 y 4 ciclos, respectivamente. Por tanto, es poco fiable afirmar que los picos espectrales ca. 2.1 y 1.3 Ma representan eventos periódicos reales. Para poderlo afirmar con mas confianza sería necesario disponer de más datos (muestras).

La ausencia del ciclo orbital de $400 \mathrm{Ka}$ en los resultados del análisis espectral (Figura 3 A y 3 B) para ambas series temporales (valores medios de $\delta^{18} \mathrm{O}$ y $\delta^{13} \mathrm{C}$ ), en principio podría deberse a la irregularidad en el muestreo y a los errores de datación. Sin embargo, estas causas pueden ser descartadas porque en el análisis espectral se tienen en cuenta estos factores. La razón más plausible de la ausencia del ciclo de $400 \mathrm{Ka}$ son las pocas muestras de belemnites $(\mathrm{N}=81)$ y la longitud de la sección estratigráfica (118 m). Por ejemplo, en el trabajo de Olsen \& Kent $(1996,1999)$, que cubre una buena parte del Jurásico Inferior, hay clara evidencia geológica de la existencia del ciclo orbital de $400 \mathrm{Ka}$, pero la resolución (espacio-temporal) estratigráfica de sus datos es de $1 \mathrm{~m} / 2 \mathrm{Ka} \mathrm{y}$ con una longitud de la sección estratigráfica de casi $5 \mathrm{~km}$, por lo cual cubre unos $35 \mathrm{Ma}$.

Utilizando los resultados del análisis espectral pero enfocados en los picos espectrales de más corto periodo se puede observar (Figura $3 \mathrm{~B}$ ) la existencia de un pico espectral significativo (robusto al ruido de fondo y a los errores de datación) en el espectro del $\delta^{13} \mathrm{C}$ localizado $c a .109 \mathrm{Ka}$. Este pico espectral podría estar relacionado con alguno de los ciclos de corto periodo de la excentricidad de la Tierra, cuya periodicidad promedio es $100 \mathrm{Ka}$ (Berger \& Loutre, 2004; Crucifix et al., 2006; Laskar et al., 2004, 2011). Desde el punto de vista de la evidencia geológica, existen varios trabajos para el Jurásico Inferior 
en los cuales se utilizan diversos registros geológicos procedentes de diferentes regiones geográficas donde han localizado el ciclo promedio de la excentricidad de $100 \mathrm{Ka}$ (Olsen, 1986; Weedon 1989; Olsen \& Kent, 1996; Hinnov \& Park, 1999; Weedon et al., 1999; Weedon \& Jenkyns, 1999; Suan et al., 2008; Kemp et al., 2011; Storm et al., 2020). Sin embargo, este ciclo no está presente en el espectro del $\delta^{18} \mathrm{O}$ (Figura $3 \mathrm{~A}$ ), donde en principio, se esperaría encontrarlo. Sin embargo, esto puede explicarse debido a que estos dos isótopos no dependen de las mismas causas. Mientras que el $\delta^{18} \mathrm{O}$ está relacionado con temperaturas del agua (e.g., circulación oceánica y paleogeografía, cambios climáticos, tipo y ubicación de los belemnites) el $\delta^{13} \mathrm{C}$ está relacionado con la productividad, tanto orgánica como inorgánica y ésta a su vez con los ciclos orbitales (Rosales et al., 2001, 2004a, 2004b, 2006).

Si observamos con más detalle la Figura 3 A, utilizando el eje de las frecuencias como eje de simetría, es posible observar una región, casi a la mitad de este eje, con altos valores de las amplitudes espectrales. El máximo pico espectral de esta región corresponde a $125 \mathrm{Ka}$. Esto podría llevar a pensar que representan un mismo evento. Sin embargo, si tenemos en cuenta el error de la localización de las frecuencias (tanto debido a los errores de datación como a la técnica utilizada para el análisis espectral), no es posible conciliar estas diferencias.

Otra posible explicación sobre el origen del pico espectral de $109 \mathrm{Ka}$ es que podría deberse a un efecto de aliasing. Se sabe por otros estudios de series temporales paleoclimáticas (Pisias \& Mix, 1988; Wunsch \& Gunn, 2003) que ciclos de más corto periodo, pueden producir picos espectrales significativos de más largo periodo, si la resolución espacial de las muestras es demasiado ancha/grande para resolver las señales de corto periodo. Por ejemplo, Pisias \& Mix (1988) demostraron que los ciclos de precesión (ca. 19 Ka y $23 \mathrm{Ka}$, valores en el presente) pueden producir picos espectrales significativos de más largo periodo cercanos a $100 \mathrm{Ka}$ cuando se utiliza un intervalo de muestreo de $25 \mathrm{Ka}$. Lo cuál podría relacionarse erróneamente con el ciclo de 100 Ka de la excentricidad. Si bien es cierto que el origen un tanto aleatorio de las localizaciones espacio-temporales de las muestras de belemnites puede reducir el fenómeno del aliasing, también es cierto que no puede evitarlo por completo (Pisias \& Mix, 1988; Eyer \& Bartholdi, 1999; Van Dongen et al., 1999). Esta hipótesis se ve reforzada, si tenemos en cuenta el ciclo de $20 \mathrm{Ka}$, relacionado con la precesión, y encontrado por Berreteaga et al. (2002) al utilizar registros de $\mathrm{CaCO}_{3}$ provenientes de la $\mathrm{BCB}$ para el Toarciense Inferior.

Para discernir si el pico espectral de $109 \mathrm{Ka}$ es una aliasing de la precesión o si podría estar relacionado con uno de los ciclos de corto periodo de la excentricidad, se ha realizado el siguiente experimento numérico. Se simularon series sintéticas equiespaciadas cada 1000 años con una señal de $21 \mathrm{Ka}$ y otra de $100 \mathrm{Ka}$ con diferentes niveles de ruido Gaussiano utilizando como referencia la media y la desviación estándar del $\delta^{13} \mathrm{C}$. Se usaron sólo los muestreos experimentales (edades) de los belemnites al estimar sus espectros (ver Figuras 4 y 5). Nuestros resultados muestran que los espectros de las series sintéticas con una señal de $21 \mathrm{Ka}$ no presentan un efecto de aliasing ca. $100 \mathrm{Ka}$ (Figura 4). Sin embargo, los espectros de las series con una señal de $100 \mathrm{Ka}$ si contienen una señal alrededor de los $100 \mathrm{Ka}$ (Figura 5). Por todo esto, podemos sostener que el pico espectral de $109 \mathrm{Ka}$ del $\delta^{13} \mathrm{C}$ no es debido a un efecto de aliasing y que este pico podría estar relacionado con los ciclos de corto periodo de la excentricidad de la Tierra.

\section{Conclusiones}

El caso de estudio presentado en este trabajo realiza por primera vez una búsqueda de señal orbital en series temporales de los valores medios de $\delta^{18} \mathrm{O}$ y $\delta^{13} \mathrm{C}$ de las rostra de belemnites de la cuenca Vasco-Cantábrica del Jurásico Inferior, para lo cual se utiliza una técnica avanzada de análisis espectral, conocida como el periodograma suavizado de Lomb-Scargle, y se tiene en cuenta los errores de datación de las muestras de belemnites en el espectro. En base a los resultados obtenidos se presentan a continuación las principales conclusiones.

En primer lugar, se concluye que no se detectaron ciclos orbitales de largo periodo, en particular, el ciclo de los $400 \mathrm{Ka}$ que se esperaba encontrar debido a que se ha mantenido estable en los últimos $250 \mathrm{Ma}$. En 
Ciclo $21 \mathrm{Ka}$. Nivel ruido 0

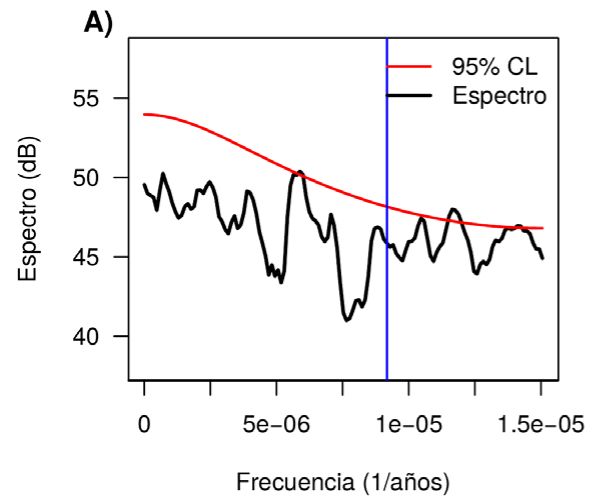

Ciclo $21 \mathrm{Ka}$. Nivel ruido 0.5

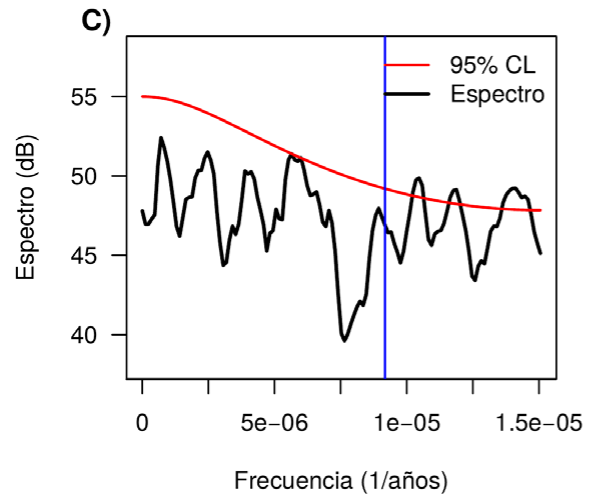

Ciclo $21 \mathrm{Ka}$. Nivel ruido 0.25

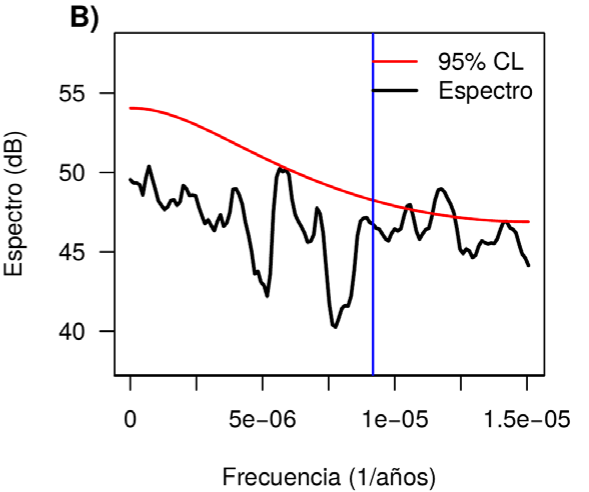

Ciclo $21 \mathrm{Ka}$. Nivel ruido 1

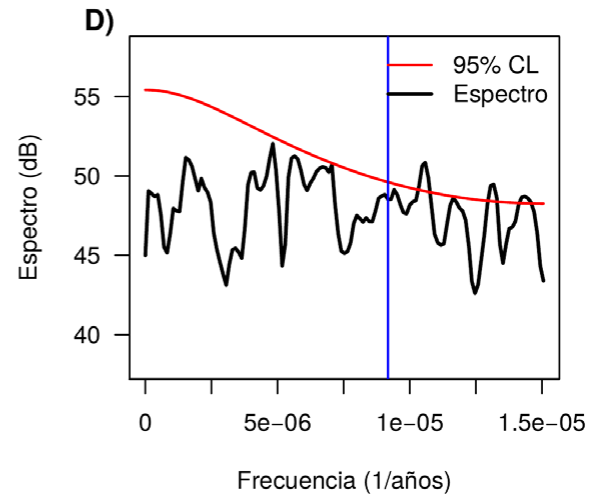

Figura 4- Espectros corregidos y suavizados (con 8 grados de libertad) de las series sintéticas con los tiempos de muestreo de los belemnites de la BCB y con una señal de $21 \mathrm{Ka}$ y con diferentes niveles de ruido Gaussiano: A) sin ruido, B), C) y D) con un 0.25 , 0.5 y 1 de la desviación estándar de los valores del $\delta^{13} \mathrm{C}$. La media del ruido Gaussiano es la media de los valores del $\delta^{13} \mathrm{C}$. La curva sólida de color rojo representa el $95 \%$ del nivel de confianza correspondiente a ruido rojo. La línea vertical azul señala la frecuencia que corresponde al pico espectral de $109 \mathrm{Ka}$. El espectro fue calculado por medio del programa REDFIT, los parámetros de entrada pueden consultarse en la Tabla 1. Fuente: elaboración propia.

segundo lugar, se concluye que el pico de $109 \mathrm{Ka}$ del espectro del $\delta^{13} \mathrm{C}$ está probablemente relacionado con los ciclos de corto periodo de la excentricidad de la Tierra y no parece que sea un efecto de aliasing como resultado de ciclos de más corto periodo, como el ciclo de la precesión (20/21 Ka). Sin embargo, se recomienda la adquisición de más muestras de belemnites de la cuenca Vasco-Cantábrica para llegar a conclusiones mucho más definitivas. Por último se concluye que la incertidumbre de la estimación espectral al considerar los errores de datación de las muestras de los belemnites aumenta en las bajas frecuencias.

Debido a los prometedores resultados obtenidos en este trabajo, se propone el uso de isótopos estables, como los valores medios de $\delta^{18} \mathrm{O}$ o de $\delta^{13} \mathrm{C}$, obtenidos de la rostra fosilizada de belemnites para ser analizados en el dominio de la frecuencia en la búsqueda de señal orbital. Algo que hasta donde sabemos no se había realizado previamente. El entendimiento de los forzamientos externos del sistema climático podría, por ejemplo, a ayudar a entender mejor el clima del Jurásico. La metodología utilizada para analizar las series temporales de valores medios de isótopos estables provenientes de macrofósiles de la cuenca Vasco-Cantábrica del Jurásico Inferior también se puede usar para analizar otros tipos de paleoindicadores ambientales provenientes de otros tipos de macrofósiles, incluso para otros tipos de datos que tengan características similares. 

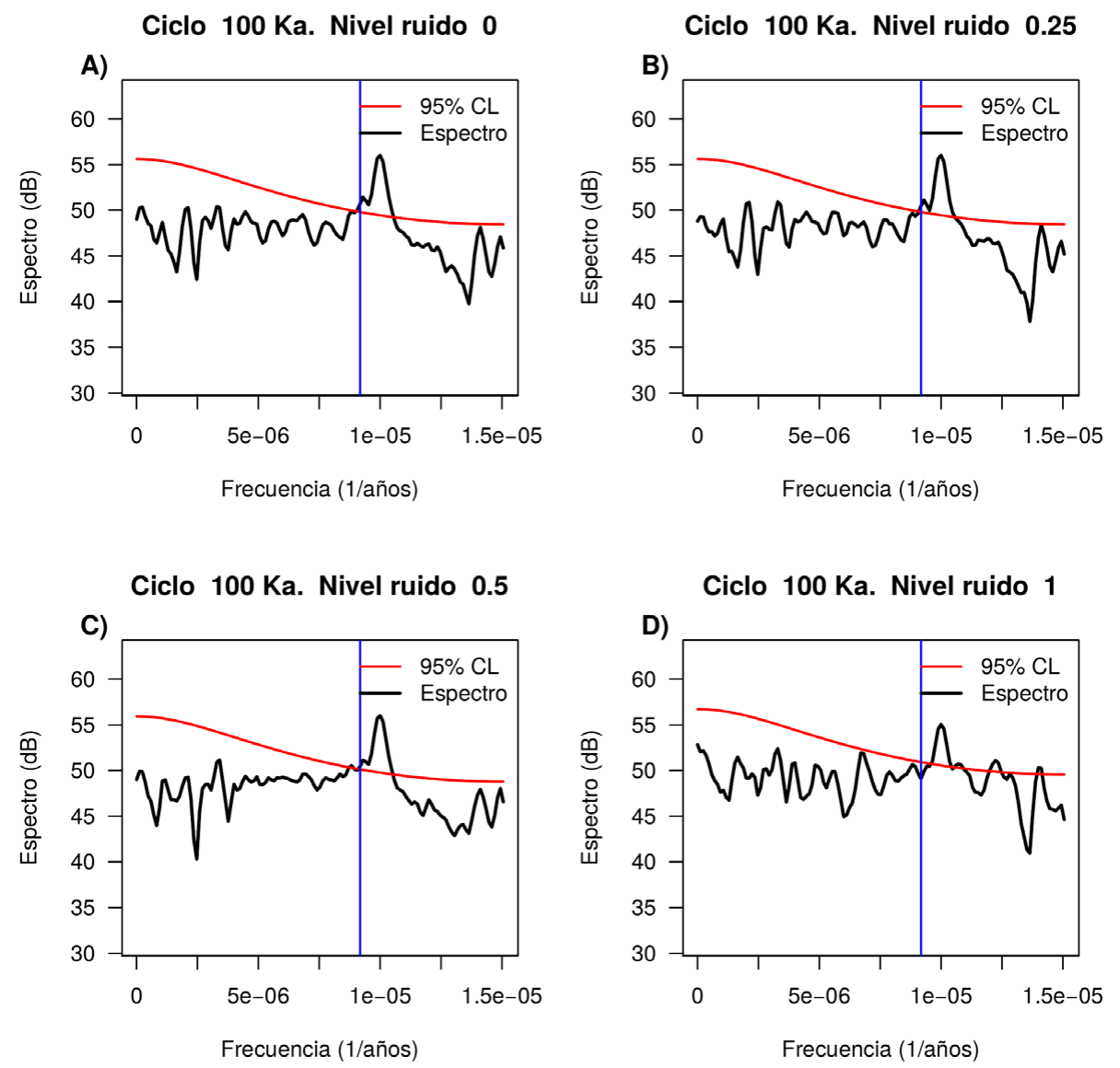

Figura 5-Espectros corregidos y suavizados (con 8 grados de libertad) de las series sintéticas con los tiempos de muestreo de los belemnites de la BCB y con una señal de $100 \mathrm{Ka}$ y con diferentes niveles de ruido Gaussiano: A) sin ruido, B), C) y D) con un 0.25 , 0.5 y 1 de la desviación estándar de los valores del $\delta^{13} \mathrm{C}$. La media del ruido Gaussiano es la media de los valores del $\delta^{13} \mathrm{C}$. La curva sólida de color rojo representa el $95 \%$ del nivel de confianza correspondiente a ruido rojo. La línea vertical azul señala la frecuencia que corresponde al pico espectral de $109 \mathrm{Ka}$. El espectro fue calculado por medio del programa REDFIT, los parámetros de entrada pueden consultarse en la Tabla 1. Fuente: elaboración propia.

\section{AGRADECIMIENTOS}

Se agradece a Idoia Rosales y Sergio Robles por proporcionar los datos usados y a Jon Saénz por sus comentarios a las primeras versiones de este trabajo. Especial agradecimiento a los revisores de este artículo Alejandro Gómez Dacal y Eulogio Pardo-Igúzquiza por sus comentarios y sugerencias que han hecho mejorar enormemente este trabajo. Finalmente, al Sistema Español Estatal de Empleo (SEPE) por la financiación en esta última etapa de este trabajo.

\section{Referencias}

Anderson, T.F.; Popp, B.N.; Williams, A.C.; Ho, L.Z. \& Hudson, J.D. (1994). The stable isotopic records of fossils from the Peterborough Member, Oxford Clay Formation (Jurassic), UK: palaeoenvironmental im- plications. Journal of the Geological Society, 151 (1): 125-138. https://doi.org/10.1144/gsjgs.151.1.0125

Arabas, A.; Schlögl, J. \& Meister, C. (2017). Early Jurassic carbon and oxygen isotope records and seawater temperature variations: Insights from marine carbonate and belemnite rostra (Pieniny Klippen Belt, Carpathians). Palaeogeography, Palaeoclimatology, Palaeoecology, 485: 119-135. https://doi.org/10.1016/j. palaeo.2017.06.007

Armendáriz, M.; Rosales, I.; Bádenas, B.; Pinuela, L.; Aurell, M. \& García-Ramos, J.C. (2013). An approach to estimate Lower Jurassic seawater oxygen-isotope composition using $\delta 18 \mathrm{O}$ and $\mathrm{Mg} / \mathrm{Ca}$ ratios of belemnite calcites (Early Pliensbachian, northern Spain). Terra Nova, 25 (6): 439-445. https://doi.org/10.1111/ ter. 12054 
Bádenas, B.; Aurell, M.; Armendáriz, M., Rosales, I.; García-Ramos, J.C. \& Piñuela, L. (2012). Sedimentary and chemostratigraphic record of climatic cycles in Lower Pliensbachian marl-limestone platform successions of Asturias (North Spain). Sedimentary Geology, 281: 119138. https://doi.org/10.1016/j.sedgeo.2012.08.010

Bailey, T.R.; Rosenthal, Y.; McArthur, J.M.; Van de Schootbrugge, B. \& Thirlwall, M.F. (2003). Paleoceanographic changes of the Late Pliensbachian-Early Toarcian interval: a possible link to the genesis of an Oceanic Anoxic Event. Earth and Planetary Science Letters, 212(3-4): 307-320. https://doi.org/10.1016/ S0012-821X(03)00278-4

Baudin, F.; Herbin, J.P.; Bassoullet, J.P.; Dercourt, J.; Lachkar, G.; Manivit, H. \& Renard, M. (1990). Distribution of organic matter during the Toarcian in the Mediterranean Tethys and Middle East. American Association of Petroleum Geologists, Tulsa, Oklahoma, USA. https://doi.org/10.1306/St30517C6

Benito, M.I.; Reolid, M. \& Viedma, C. (2016). On the microstructure, growth pattern and original porosity of belemnite rostra: insights from calcitic Jurassic belemnites. Journal of Iberian Geology, 42 (2): 201-226.

Berger, A. \& Loutre, M.F. (2004). Astronomical theory of climate change. Journal of Physics IV France, 121: 1-35. https://doi.org/10.1051/jp4:2004121001

Berreteaga, A.; Robles, S.; Rosales, I. \& Quesada, S. (2002). Ciclos de precesión en el Toarciense de la Cuenca Vasco-Cantábrica. Geogaceta, 32: 259-262.

Blaauw, M. (2012). Out of tune: the dangers of aligning proxy archives. Quaternary Science Reviews, 36: 3849. https://doi.org/10.1016/j.quascirev.2010.11.012

Bodin, S.; Krencker, F.N.; Kothe, T.; Hoffmann, R.; Mattioli, E.; Heimhofer, U. \& Kabiri, L. (2016). Perturbation of the carbon cycle during the late Pliensbachian-early Toarcian: New insight from high-resolution carbon isotope records in Morocco. Journal of African Earth Sciences, 116: 89-104. https://doi.org/10.1016/j.jafrearsci.2015.12.018

Braga, J.C.; Comas-Rengifo, M.J.; Goy, A.; Rivas, P. \& Yebenes, A. (1988). El lias inferior y medio en zona central de la Cuenca Vasco-Cantabrica (Camino, Santander). III Coloquio de Estratigrafía y Paleontología del Jurásico de España, Logroño, 17-43.

Comas-Rengifo, M.J.; Goy, A.; Rivas, P. \& Yebenes, A. (1988). El Toarciense en Castillo Pedroso (Santander). III Coloquio de Estratigrafía y Paleontología del Jurásico de España, Logroño, 63-71.

Crucifix, M.; Loutre, M.-F. \& Berger, A. (2006). The climate response to the astronomical forcing. Space Science Reviews, 125 (1-4): 213-226. https://doi. org/10.1007/s11214-006-9058-1
Decourt, J.; Ricou, L.E. \& Vrielynck, B. (1993). Atlas Tethys Palaeoenvironmental Maps. Gauthier-Vollars, Paris, France.

Doyle, P. \& MacDonald, D.I.M. (1993). Belemnite battlefields. Lethaia, 26 (1): 65-80. https://doi.org/10.1111/j.1502-3931.1993.tb01513.x

Eyer, L. \& Bartholdi, P. (1999). Variable stars: which Nyquist frequency? Astronomy and Astrophysics, Supplement Series, 135 (1): 1-3. https://doi.org/10.1051/ aas:1999102

Fleitmann, D.; Burns, S.J.; Mudelsee, M.; Neff, U.; Kramers, J.; Mangini, A. \& Matter, A. (2003). Holocene forcing of the Indian monsoon recorded in a stalagmite from Southern Oman. Science, 300 (5626): 17371739. https://doi.org/10.1126/science. 1083130

Gilman, D.L.; Fuglister, F.J. \& Mitchell Jr, J.M. (1963). On the power spectrum of "red noise". Journal of Atmospheric Sciences, 20 (2): 182-184. https://doi.or$\mathrm{g} / 10.1175 / 1520-0469(1963) 020<0182$ :OTPSON $>2.0$. $\mathrm{CO} ; 2$

Gómez, J.J.; Goy, A. \& Canales, M.L. (2008). Seawater temperature and carbon isotope variations in belemnites linked to mass extinction during the Toarcian (Early Jurassic) in central and Northern Spain. Comparison with other European sections. Palaeogeography, Palaeoclimatology, Palaeoecology, 258 (1-2): 28-58. https://doi.org/10.1016/j.palaeo.2007.11.005

Gómez, J.J.; Comas-Rengifo, M.J. \& Goy, A. (2016). Palaeoclimatic oscillations in the Pliensbachian (early Jurassic) of the Asturian Basin (northern Spain). Climate of the Past, 12 (5): 1199-1214. https://doi. org/10.5194/cp-12-1199-2016

Hasselmann, K. (1976). Stochastic climate models Part I. Theory. Tellus, 28 (6): 473-485. https://doi. org/10.3402/tellusa.v28i6.11316

Hermoso, M.; Minoletti, F.; Rickaby, R.E.; Hesselbo, S.P.; Baudin, F. \& Jenkyns, H.C. (2012). Dynamics of a stepped carbon-isotope excursion: Ultra high-resolution study of Early Toarcian environmental change. Earth and Planetary Science Letters, 319: 45-54. https://doi. org/10.1016/j.epsl.2011.12.021

Hesselbo, S. P.; Gröcke, D.R.; Jenkyns, H.C.; Bjerrum, C.J.; Farrimond, P.; Bell, H.S. M. \& Green, O.R. (2000). Massive dissociation of gas hydrate during a Jurassic oceanic anoxic event. Nature, 406(6794): 392-395. https://doi.org/10.1038/35019044

Hinnov, L.A. \& Park, J.F. (1999). Strategies for assessing Early-Middle (Pliensbachian-Aalenian)-Jurassic cyclochronologies. Philosophical Transactions of the Royal Society A, 357: 1831-1859. https://doi. org/10.1098/rsta.1999.0403 
Hinnov, L.A. \& Ogg, J.G. (2007). Cyclostratigraphy and the Astronomical Time Scale. Stratigraphy, 4 (2-3): 239-251.

Hoffmann, R. \& Stevens, K. (2020). The palaeobiology of belemnites-foundation for the interpretation of rostrum geochemistry. Biological Reviews, 95 (1): 94123. https://doi.org/10.1111/brv. 12557

Huybers, P.J. \& Wunsch, C. (2004). A Depth-Derived Pleistocene Age-Model: Uncertainty Estimates, Sedimentation Variability, and Nonlinear Climate Change. Paleoceanography, 19: 1-24. https://doi.or$\mathrm{g} / 10.1029 / 2002 \mathrm{PA} 000857$

Immenhauser, A.; Schoene, B.R.; Hoffmann, R. \& Niedermayr, A. (2016). Mollusc and brachiopod skeletal hard parts: intricate archives of their marine environment. Sedimentology, 63(1): 1-59. https://doi.org/10.1111/ sed. 12231

Jenkyns, H.C.; Jones, C.E.; Grocke, D.R.; Hesselbo, S.P. \& Parkinson, D.N. (2002). Chemostratigraphy of the Jurassic System: applications, limitations and implications for palaeoceanography. Journal of the Geological Society, 159 (4): 351-378. https://doi. org/10.1144/0016-764901-130

Kemp, D.B.; Coe, A.L.; Cohen, A.S. \& Schwark, L. (2005). Astronomical pacing of methane release in the Early Jurassic period. Nature, 437(7057): 396-399. https://doi.org/10.1038/nature04037

Kemp, D.B.; Coe, A.L., Cohen, A.S. \& Weedon, G.P. (2011). Astronomical forcing and chronology of the early Toarcian (Early Jurassic) oceanic anoxic event in Yorkshire, UK. Paleoceanography, 26 (4): PA4210. https://doi.org/10.1029/2011PA002122

Kemp, D.B. \& Izumi, K. (2014). Multiproxy geochemical analysis of a Panthalassic margin record of the early Toarcian oceanic anoxic event (Toyora area, Japan). Palaeogeography, Palaeoclimatology, Palaeoecology, 414: 332-341. https://doi.org/10.1016/j. palaeo.2014.09.019

Laskar, J.; Robutel, P.; Joutel, F.; Gastineau, M.; Correia, A.C.M. \& Levrard, B. (2004). A long-term numerical solution for the insolation quantities of the Earth. Astronomy \& Astrophysics, 428: 261-285. https://doi. org/10.1051/0004-6361:20041335

Laskar, J.; Fienga, A.; Gastineau, M. \& Manche, H. (2011). La2010: a new orbital solution for the long-term motion of the Earth. Astronomy \& Astrophysics, 532: A89. https://doi.org/10.1051/0004-6361/201116836

Lisiecki, L. (2005). Paleoclimate time series: New alignment and compositing techniques, a 5.3-Myr benthic $\delta 18 \mathrm{O}$ stack, and analysis of Pliocene-Plesitocene climate transitions. Ph.D. thesis, Brown University, USA, 106 pp.

Lomb, N.R. (1976). Least-squares frequency analysis of unequally spaced data. Astrophysics and Space
Sciences, 39 (2): 447-462 https://doi.org/10.1007/ BF00648343

McArthur, J.M.; Donovan, D.T.; Thirlwall, M.F.; Fouke, B.W. \& Mattey, D. (2000). Strontium isotope profile of the early Toarcian (Jurassic) oceanic anoxic event, the duration of ammonite biozones, and belemnite palaeotemperatures. Earth and Planetary Sciences Letters, 179 (2): 269-285. https://doi.org/10.1016/S0012$821 \mathrm{X}(00) 00111-4$

McArthur, J.M.; Howarth, R.J. \& Bailey, T.R. (2001). Strontium Isotope Stratigraphy: LOWESS version 3: Best Fit to the Marine Sr-Isotope Curve for 0-509 Ma and Accompanying Look-up Table for Deriving Numerical Age. The Journal of Geology, 109: 155-170. https://doi.org/10.1086/319243

McArthur, J.M.; Doyle, P.; Leng, M.J.; Reeves, K.; Williams, C.T.; Garcia-Sánchez, R. \& Howarth, R.J. (2007). Testing palaeo-environmental proxies in Jurassic belemnites: $\mathrm{Mg} / \mathrm{Ca}, \mathrm{Sr} / \mathrm{Ca}, \mathrm{Na} / \mathrm{Ca}, \delta 18 \mathrm{O}$ and 813C. Palaeogeography, Palaeoclimatology, Palaeoecology, 252 (3-4): 464-480. https://doi.org/10.1016/j. palaeo.2007.05.006

Medina-Elizalde, M.; Polanco-Martínez, J.M.; Lases-Hernández, F.; Bradley, R. \& Burns, S. (2016). Testing the "tropical storm" hypothesis of Yucatan Peninsula climate variability during the Maya Terminal Classic Period. Quaternary Research, 86 (2): 111-119. https:// doi.org/10.1016/j.yqres.2016.05.006

Mudelsee, M.; Scholz, D.; Röthlisberger, R.; Fleitmann, D.; Mangini, A. \& Wolff, E.W. (2009). Climate spectrum estimation in the presence of timescale errors. Nonlinear Processes in Geophysics, 16: 43-56. https:// doi.org/10.5194/npg-16-43-2009

Nava, A. (2010). Fourier spectral analysis for unevenly spaced, average value, data. Computers \& Geosciences, 36 (7): 853-860. https://doi.org/10.1016/j.cageo.2009.12.002

Oliveira, D.; Sánchez Goñi, M.F.; Naughton, F.; Polanco-Martínez, J.; Jimenez-Espejo, F.J.; Grimalt, J.O.; Martrat, B.; Voelker, A.H.; Trigo, R. \& Hodell, D.; Abrantes F.; \& Desprat, S. (2017). Unexpected weak seasonal climate in the western Mediterranean region during MIS 31, a high-insolation forced interglacial. Quaternary Science Reviews, 161: 1-17. https://doi. org/10.1016/j.quascirev.2017.02.013

Olsen, P.E. (1986). A 40-million-year lake record of early mesozoic orbital climatic forcing. Science, 234 (4778): 842-848. https://doi.org/10.1126/science. 234.4778 .842

Olsen, P.E. \& Kent, D.V. (1996). Milankovitch climate forcing in the tropics of Pangea during the Late Triassic. Palaeogeography, Palaeoclimatology, Palaeoeco- 
logy, 122 (1-4): 1-26. https://doi.org/10.1016/00310182(95)00171-9

Olsen, P.E. \& Kent, D.V. (1999). Long-period Milankovitch cycles from the Late Triassic and Early Jurassic of eastern North America and their implications for the calibration of the Early Mesozoic time-scale and the long-term behaviour of the planets. Philosophical Transactions of the Royal Society A, 357: 1761-1786. https://doi.org/10.1098/rsta.1999.0400

Pálfy, J.; Smith, P.L. \& Mortensen, J.K. (2000). A U-Pb and $40 \mathrm{Ar} / 39 \mathrm{Ar}$ time scale for the Jurassic. Canadian Journal of Earth Sciences, 37 (6): 923-944. https://doi. org/10.1139/e00-002

Pardo-Iguzquiza, E. \& Rodríguez-Tovar, F.J. (2011). Implemented Lomb-Scargle periodogram: a valuable tool for improving cyclostratigraphic research on unevenly sampled deep-sea stratigraphic sequences. Geo-Marine Letters, 31(5): 537-545. https://doi.org/10.1007/ s00367-011-0247-x

Pardo-Igúzquiza, E. \& Rodríguez-Tovar, F.J. (2012). Spectral and cross-spectral analysis of uneven time series with the smoothed Lomb-Scargle periodogram and Monte Carlo evaluation of statistical significance. Computers \& Geosciences, 49: 207-216. https://doi. org/10.1016/j.cageo.2012.06.018

Pardo-Igúzquiza E. \& Rodríguez-Tovar F.J. (2013). Análisis espectral de series temporales de variables geológicas con muestreo irregular. Boletín Geológico y Minero, 124 (3): 323-337.

Pisias, N.G. \& Mix, A.C. (1988). Aliasing of the geologic record and the search for long-period Milankovitch cycles. Paleoceanography, 3 (5): 613-619. https://doi. org/10.1029/PA003i005p00613

Podlaha, O.G.; Mutterlose, J. \& Veizer, J. (1998). Preservation of $\delta 18 \mathrm{O}$ and $\delta 13 \mathrm{C}$ in belemnite rostra from the Jurassic/Early Cretaceous successions. Americal Journal of Sciences, 298 (4): 324-347. https://doi. org/10.2475/ajs.298.4.324

Polanco-Martínez, J.M. (2012). Aplicación de técnicas estadísticas en el estudio de fenómenos ambientales y ecosistémicos. Tesis doctoral, Universidad del País Vasco, 208 pp.

Polanco-Martínez, J.M. (2014). Estimación espectral de datos ambientales no equiespaciados vía el periodograma suavizado de Lomb-Scargle. Una breve revisión. Analítika: revista de análisis estadístico, 8 (2): 7-23.

Polanco-Martínez, J.M. \& Faria, S. (2018). Estimation of the significance of the Foster's wavelet spectrum by means of a permutation test and its application for paleoclimate records. Boletín Geológico y Minero, 129 (3): 549-564. https://doi.org/10.21701/bolgeo$\min .129 .3 .006$
Price, G.D. \& Sellwood, B.W. (1997). Warm palaeotemperatures from high Late Jurassic palaeolatitudes (Falkland Plateau): Ecological, environmental or diagenetic controls? Palaeogeography, Palaeoclimatology, Palaeoecology, 129 (3): 315-328. https://doi. org/10.1016/S0031-0182(96)00058-2

Remírez, M.N. \& Algeo, T.J. (2020). Carbon-cycle changes during the Toarcian (Early Jurassic) and implications for regional versus global drivers of the Toarcian oceanic anoxic event. Earth-Science Reviews, 209: 103283. https://doi.org/10.1016/j.earscirev.2020.103283

Rexfort, A. \& Mutterlose, J. (2006). Stable isotope records from Sepia officinalis - a key to understanding the ecology of belemnites? Earth and Planetary Sciences Letters, 30: 212-221. https://doi.org/10.1016/j. eps1.2006.04.025

Rosales, I.; Quesada, S. \& Robles, S. (2001). Primary and diagenetic isotopic signals in fossils and hemipelagic carbonates: the Lower Jurassic of northern Spain. Sedimentology, 48 (5): 1149-1169. https://doi. org/10.1046/j.1365-3091.2001.00412.x

Rosales, I.; Quesada, S. \& Robles, S. (2004a). Paleotemperature variations of Early Jurassic seawater recorded in geochemical trends of belemnites from the Basque-Cantabrian basin, northern Spain. Palaeogeography, Palaeoclimatology, Palaeoecology, 203: 253275. https://doi.org/10.1016/S0031-0182(03)00686-2

Rosales, I.; Robles, S. \& Quesada, S. (2004b). Elemental and oxygen isotope composition of Early Jurassic belemnites: salinity vs. temperature signals. Journal of Sedimentary Research, 74 (3): 342-354. https://doi. org/10.1306/112603740342

Rosales, I.; Quesada, S. \& Robles, S. (2006). Geochemical arguments for identifying second-order sea-level changes in hemipelagic carbonate ramp deposits. Terra Nova, 18 (4): 233-240. https://doi.org/10.1111/j.13653121.2006.00684.x

Sælen, G.; Doyle, P. \& Talbot, M.R. (1996). Stable-isotope analyses of belemnite rostra from the Whitby Mudstone, England: surface water conditions during deposition of a marine black shale. Palaios, 11 (2): 97117. https://doi.org/10.2307/3515065

Scargle, J. D. (1982). Studies in astronomical time series analysis. II- Statistical aspects of spectral analysis of unevenly spaced data. The Astrophysical Journal, 263: 835-853. https://doi.org/10.1086/160554

Scargle, J.D. (1989). Studies in astronomical time series analysis. III- Fourier transforms, autocorrelation functions, and cross-correlation functions of unevenly spaced data. The Astrophysical Journal, 343: 874-887. https://doi.org/10.1086/167757 
Schulz, M. \& Mudelsee, M. (2002). REDFIT: Estimating red-noise spectra directly from unevenly spaced paleoclimatic time series. Computers \& Geosciences, 28 (3): 421-426. https://doi.org/10.1016/S00983004(01)00044-9

Schulz, M. \& Stattegger, K. (1997). SPECTRUM: Spectral analysis of unevenly spaced paleoclimatic time series. Computers \& Geosciences, 23 (9): 929-945. https://doi.org/10.1016/S0098-3004(97)00087-3

Storm, M.S.; Hesselbo, S.P.; Jenkyns, H.C.; Ruhl, M.; Ullmann, C.V.; Xu, W.; Leng, M.J.; Riding, J.B. \& Gorbanenko, O. (2020). Orbital pacing and secular evolution of the early jurassic carbon cycle. Proceedings of the National Academy of Sciences, 117 (8): 3974-3982. https://doi.org/10.1073/pnas.1912094117

Suan, G.; Pittet, B.; Bour, I.; Mattiolia, E.; Duarte, L.V. \& Mailliot, S. (2008). Duration of the Early Toarcian carbon isotope excursion deduced from spectral analysis: Consequence for its possible causes. Earth and Planetary Science Letters, 267 (3-4): 666-679. https://doi. org/10.1016/j.epsl.2007.12.017

Van Dongen, H.P.A.; Olofsen, E.; Van Hartevelt, J.H. \& Kruyt, E.W. (1999). A procedure of multiple period searching in unequally spaced time-series with the Lomb-Scargle method. Biological Rhythm Research, 30 (2): 149-177. https://doi.org/10.1076/ brhm.30.2.149.1424

VanderPlas, J.T. (2018). Understanding the Lomb-Scargle periodogram. The As-trophysical Journal Supplement Series, 236 (1): 16. https://doi.org/10.3847/15384365/aab766

Vio, R.; Diaz-Trigo, M. \& Andreani, P. (2013). Irregular time series in astronomy and the use of the Lomb-Scargle periodogram. Astronomy and Computing, 1: 5-16. https://doi.org/10.1016/j.ascom.2012.12.001

Weedon, G.P. (1989). The detection and illustration of re- gular sedimentary cycles using Walsh power spectra and filtering, with examples from the Lias of Switzerland. Journal of the Geological Sociery, 146 (1): 133144. https://doi.org/10.1144/gsjgs.146.1.0133

Weedon, G.P. (2003). Time-series analysis and cyclostratigraphy: examining stratigraphic records of environmental cycles. Cambridge Univ Press, Cambridge. https://doi.org/10.1017/CBO9780511535482

Weedon, G.P. \& Jenkyns, H.C. (1999). Cyclostratigraphy and the Early Jurassic timescale: Data from the Belemnite Marls, Dorset, southern England. The Geological Society of America Bulletin, 111: 1823-1840. https://doi.org/10.1130/0016-7606(1999)111<1823:CATEJT $>2.3 . \mathrm{CO} ; 2$

Weedon, G.P.; Jenkyns, H.C.; Coe, A.L. \& Hesselbo, S.P. (1999). Astronomical calibration of the Jurassic time-scale from cyclostratigraphy in British mudrock formations. Philosophical Transactions of the Royal Society A, 357: 1787-1813. https://doi.org/10.1098/rsta.1999.0401

Wierzbowski, H. (2004). Carbon and oxygen isotope composition of Oxfordian Early Kimmeridgian belemnite rostra: palaeoenvironmental implications for Late Jurassic seas. Palaeogeography, Palaeoclimatology, Palaeoecology, 203 (1-2): 153-168. https://doi. org/10.1016/S0031-0182(03)00673-4

Wu, Z.; Huang, N.E.,; Long, S.R. \& Peng C.K. (2007). On the trend, detrending, and variability of nonlinear and nonstationary time series. Proceedings of the National Academy Sciences 104, (38): 14889. https://doi. org/10.1073/pnas.0701020104

Wunsch, C. \& Gunn, D.E., 2003. A densely sampled core and climate variable aliasing. Geo-marine Letters, 23 (1): 64-71. https://doi.org/10.1007/s00367-003-0125-2

Ziegler, P. A. (1990). Geological atlas of western and central Europe. Shell Internationale Petroleum Maatschappij B.V., The Hague, 239 pp. 